\title{
CARACTERIZAÇÃO DA PRECIPITAÇÃO MENSAL, SAZONAL E ANUAL PARA O ESTADO DO PARANÁ EM PERÍODOS SECOS, NORMAIS E CHUVOSOS (1977-2006)
}

\author{
Yara Rúbia de Mello ${ }^{(a)}$, Mariana Bini Leite ${ }^{(b)}$ \\ (a) Programa de Pós-Graduação em Geografia, Universidade Federal do Paraná, yarademello@ gmail.com \\ (b) Departamento de Engenharia Ambiental e Sanitária, Universidade da Região de Joinville - Univille, \\ marianabinileite@gmail.com
}

Eixo: 3. Climatologia em diferentes níveis escalares: mudanças e variabilidades

\begin{abstract}
Resumo
O objetivo deste estudo foi classificar a precipitação mensal, sazonal e anual no Estado do Paraná, em períodos secos, normais e chuvosos (1977-2006). Para isso, foram utilizados dados de precipitação de 166 pluviômetros, e selecionados dois pluviômetros representativos de cada região homogênea, as quais foram determinadas a partir da técnica de agrupamento k-means. Posteriormente aplicou-se a técnica dos quantis para cada estação, e para a média das 166 estações, a fim de classificar os períodos em muito-secos $\left(\mathrm{Q}_{0,15}\right)$, secos $\left(\mathrm{Q}_{0,15}-\mathrm{Q}_{0,35}\right)$, normais $\left(\mathrm{Q}_{0,35}-\mathrm{Q}_{0,65}\right)$, chuvosos $\left(\mathrm{Q}_{0,65}-\mathrm{Q}_{0,85}\right)$ e muitochuvosos $\left(\mathrm{Q}_{0,85}\right)$; foram elaborados dendogramas para auxiliar nas análises. Dentre os resultados obteve-se que os anos muito-chuvosos foram 1983, 1998, 1997 e 1990; e os anos muito-secos foram 1985, 1978, 1988 e 2006. No ano de 1983, o outono, inverno e a primavera foram as estações do ano classificadas como muito-chuvosas. E em 1985, o inverno e a primavera foram as estações classificadas como muito-secas.
\end{abstract}

Palavras chave: Anos padrões; períodos padrões; quantis; dendograma; k-means.

\section{Introdução}

Entender o comportamento da precipitação pluviométrica ao longo do tempo é um conhecimento de grande valia para a sociedade. Este parâmetro meteorológico é um dos que mais influencia o cotidiano da população, em questões tais como a produção de alimentos, abastecimento público, planejamento urbano, desastres naturais e turismo.

Ao estudar uma série histórica de precipitação evidencia-se a ocorrência de valores mais frequentes ou habituais, os quais podem ser designados como normais; e valores extremos ou excepcionais, que ocorrem com menor frequência, os quais podem ser designados como períodos muito secos ou muito chuvosos (PINKAYAN, 1966; SANTOS et al.,2016).

Esta classificação das precipitações é muito utilizada na análise rítmica, onde a seleção de "períodos padrão", possibilita representar uma série histórica por meio da análise de padrões habituais e excepcionais (MONTEIRO, 1969; TAVARES, 1976; SILVA, 1999; ZANDONADI, 2009; ZAVATTINI 
e BOIN, 2013). Estudos com foco na caracterização da precipitação de uma região também utilizam de classificações da precipitação (GUIMARÃES e LOPES, 2015; SANTOS et al.,2016).

A técnica dos quantis (percentis) é um método amplamente utilizado para caracterizar a precipitação (ZANDONADI, 2009; GUIMARÃES e LOPES, 2015; SANTOS et al.,2016). Esta técnica consiste na análise da distribuição da frequência acumulada dos dados, onde são selecionadas classes (quantis) que representam as frequências ou probabilidades de ocorrência do fenômeno. Ressalva-se que são utilizados diferentes quantis na literatura, e as classes utilizadas por Pinkaian (1966) são comumente empregadas.

Estudos como os de Tavares (1976) e de Zavattini e Boin (2013) utilizaram de outra técnica para auxiliar na categorização de dados de precipitação em períodos secos, normais, e chuvosos: a análise de agrupamento (cluster analysis). Esta técnica multivariada consiste no agrupamento de um conjunto de dados a partir da similaridade entre eles.

O presente estudo objetivou a partir destas técnicas realizar uma análise classificatória da precipitação mensal, sazonal e anual do Estado do Paraná, de 1977 a 2006, em períodos secos, normais e chuvosos. Com vista a auxiliar na seleção de períodos para a avaliação da adequação de interpoladores para representar a distribuição espacial da precipitação; já que este trabalho é parte de um estudo no qual se pretende realizar um mapeamento geoestatístico da precipitação para o estado do Paraná.

\section{Materiais e Métodos}

\section{1 Área de estudo}

A área de estudo compreende o estado Paraná - PR, o qual está localizado na Região Sul do Brasil, entre as latitudes $22^{\circ} 30^{\prime} \mathrm{S}$ e $26^{\circ} 43^{\prime} \mathrm{S}$ e longitudes $48^{\circ} 05^{\prime} \mathrm{W}$ e $54^{\circ} 37^{\prime} \mathrm{W}$ (Figura 1 ).

O Clima do Paraná, segundo a classificação de Köppen, pode ser dividido em Cfa (Clima subtropical com tendência de concentração das chuvas nos meses de verão, sem estação seca definida) na porção norte, oeste, grande parte do sudoeste e parte da região sul do Estado; e Cfb (Clima temperado sem estação seca definida) na região sul do PR (FRITZSONS, 2011). 


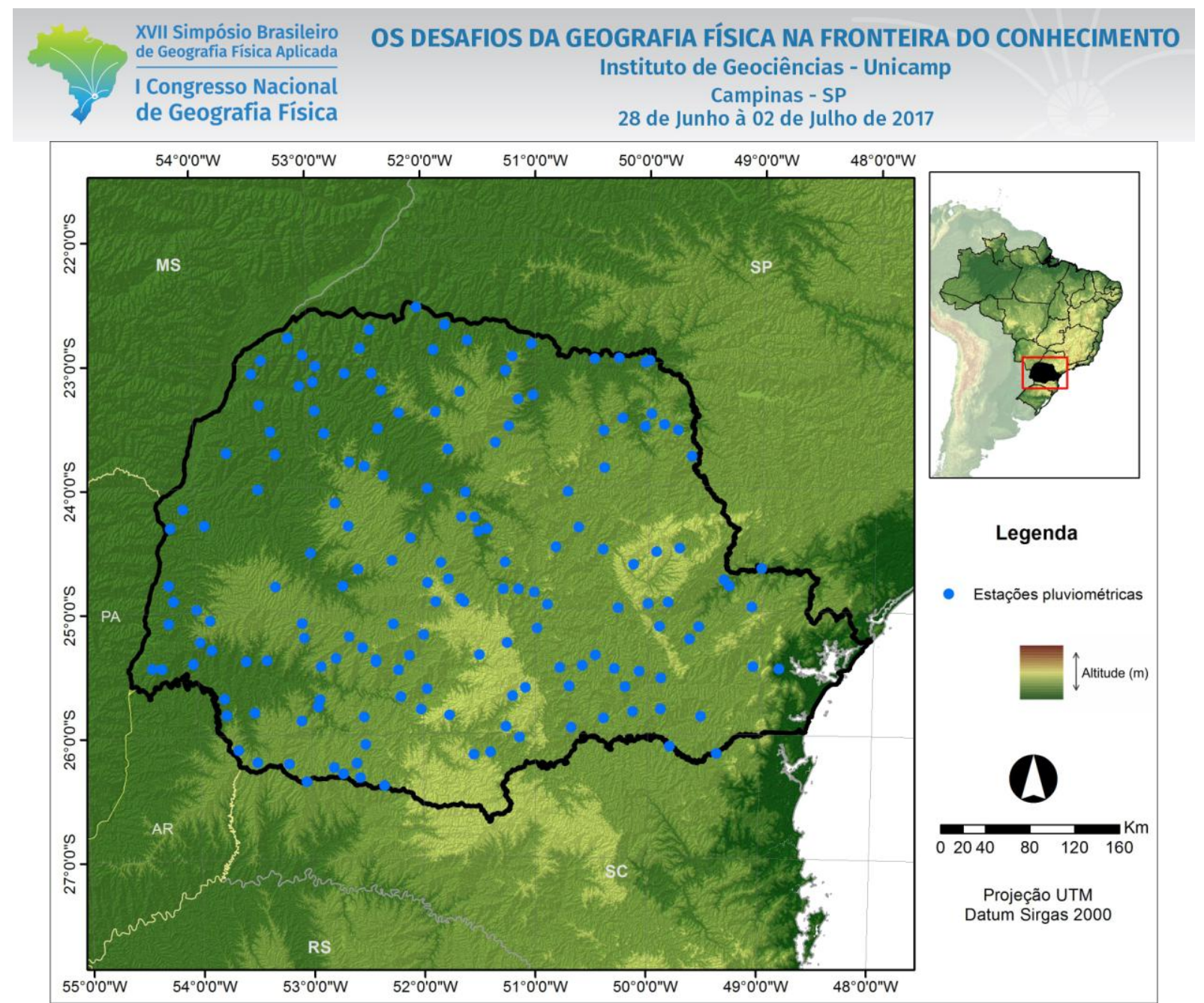

Figura 1 - Localização do Estado do Paraná e das 166 estações pluviométricas utilizadas na pesquisa. Fonte: ANA; IBGE; SRTM90.

\subsection{Estações pluviométricas}

Foram utilizados os dados de precipitação das 166 estações pluviométricas disponíveis no site da Agência Nacional de Águas, por meio do link: hidroweb.ana.gov.br, que não apresentavam falhas para o período de 1977 a 2006 no estado do Paraná (Figura 1).

Em seguida foi realizada a análise de agrupamento não hierárquica $k$-means no software Statistica 10, com a média anual das 166 estações pluviométricas, dividindo-as em quatro grupos. Cada grupo representou uma região homogênea de precipitação. Com as estações agrupadas, foram selecionadas duas estações para representar cada grupo; a seleção das estações se baseou nos menores valores de desvio percentual (diferença em porcentagem entre a média da estação e a média de todas as estações da região homogênea), e da localização geográfica da estação. Foram selecionadas estações de modo a representar as diferenças do relevo estadual. 


\subsection{Análises estatísticas}

Com os valores de precipitação das estações selecionadas para cada grupo e da média geral das 166 estações, foi efetuada a análise dos quantis. Os quantis utilizados foram os seguintes:

Tabela 1 - Intervalos de classe para classificação da precipitação.

\begin{tabular}{ccc}
\hline Probabilidade & Categoria & Quantificação (\%) \\
\hline $\mathrm{P}<\mathrm{Q}_{0,15}$ & Muito Seco "MS" & 15 \\
$\mathrm{Q}_{0,15} \leq \mathrm{P}<\mathrm{Q}_{0,35}$ & Seco "S" & 20 \\
$\mathrm{Q}_{0,35} \leq \mathrm{P}<\mathrm{Q}_{0,65}$ & Normal "N" & 30 \\
$\mathrm{Q}_{0,65} \leq \mathrm{P}<\mathrm{Q}_{0,85}$ & Chuvoso "C" & 20 \\
$\mathrm{P}>\mathrm{Q}_{0,85}$ & Muito Chuvoso "MC" & 15 \\
\hline \multicolumn{2}{r}{ Fonte: PINKAYAN, 1966; SANTOS et al.,2016. }
\end{tabular}

Para auxiliar na avaliação dos resultados de cada estação e da média geral, foi realizada a análise de agrupamento hierárquica denominada diagrama de árvore ou dendograma, para todos os períodos de análise. Utilizou-se o software Statistica 10, o método de ligação "single linkage" e a medida de distância "euclidian distances".

Por fim, foi elaborado um quadro síntese com base nos dados de todas as estações e da média.

\section{Resultados e Discussão}

Por meio da Figura 2, é possível observar como se deu a divisão dos quatro grupos homogêneos de estações. Na mesma figura estão destacadas as estações utilizadas como representativas de cada região homogênea, as quais estão descritas na Tabela 2. No grupo 1 (menor precipitação média) houve um total de 47 estações; no grupo 2 um total de 46 estações; no grupo 3, um total de 36 estações; e no grupo 4 (maior precipitação média), um total de 37 estações.

Com estas estações representativas de cada região foram analisados os quantis (muito seco $\left(\mathrm{Q}_{0,15}\right)$, seco $\left(\mathrm{Q}_{0,15}-\mathrm{Q}_{0,35}\right)$, normal $\left(\mathrm{Q}_{0,35}-\mathrm{Q}_{0,65}\right)$, chuvoso $\left(\mathrm{Q}_{0,65}-\mathrm{Q}_{0,85}\right)$ e muito chuvoso $\left.\left(\mathrm{Q}_{0,85}\right)\right)$ referentes aos dados de precipitação de cada período (ex.: janeiro, fevereiro, verão, primavera, anual, etc.), para cada estação separadamente; e também para a média das 166 estações. Com a avaliação destes resultados, e dos dendogramas confeccionados para cada período, foram gerados os quadros síntese (Figuras 3 e 4), que serão discutidos a seguir. 


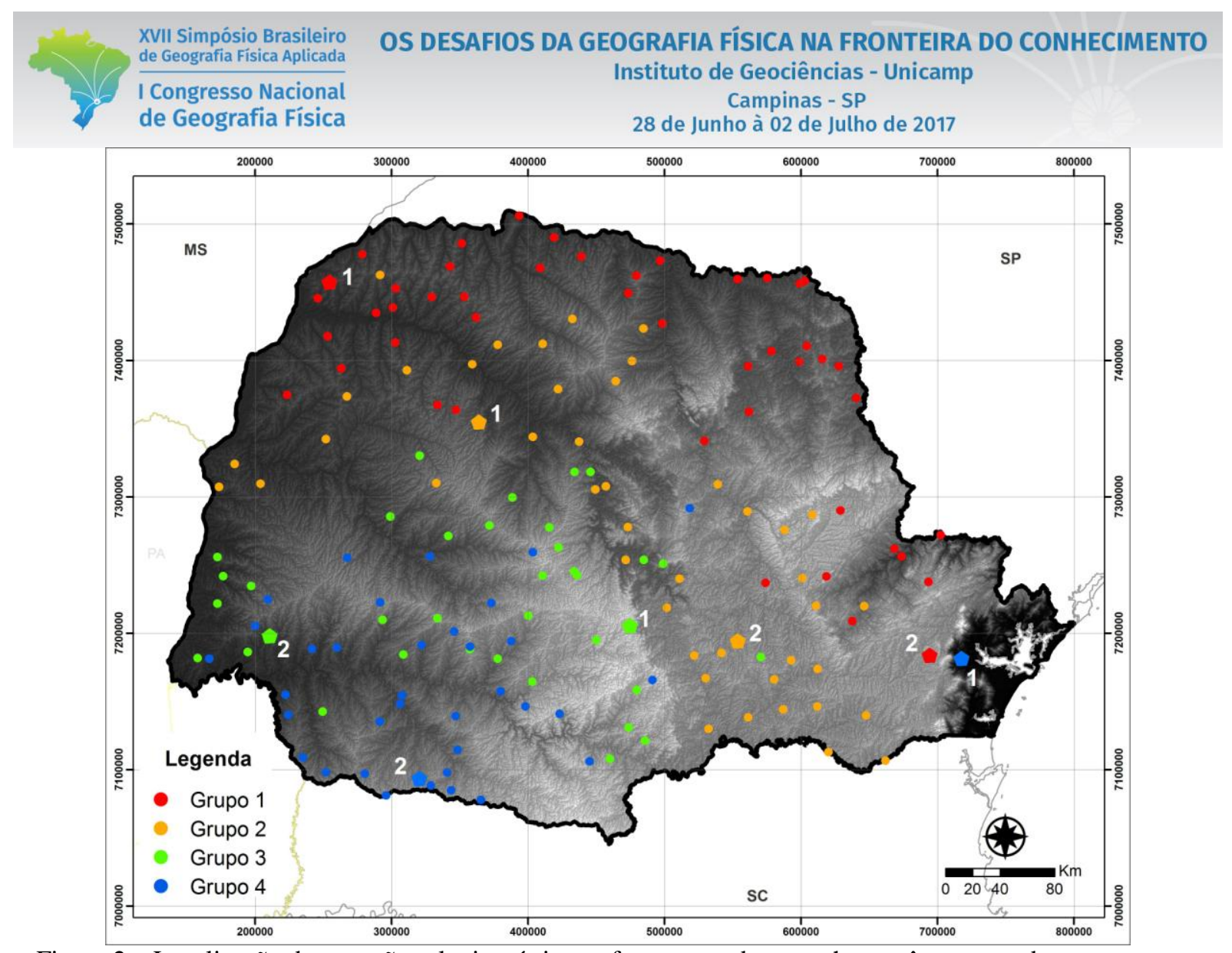

Figura 2 - Localização das estações pluviométricas referente a cada grupo homogêneo, com destaque para as estações representativas de cada grupo. Fonte: ANA; IBGE; SRTM90.

Tabela 2 - Descrição das estações pluviométricas representativas dos grupos homogêneos.

\begin{tabular}{ccccccccc}
\hline & Média/Grupo & Contagem & Código & Nome & Altitude & Média & Mínimo & Máximo \\
\hline \multirow{2}{*}{ GRUPO 1 } & \multirow{2}{*}{1401,2} & 1 & 2253010 & ICATU & 399 & 1395,6 & 917,5 & 1909,3 \\
& & 2 & 2549004 & PIRAQUARA & 900 & 1396,5 & 738,6 & 2062,3 \\
\multirow{2}{*}{ GRUPO 2 } & \multirow{2}{*}{1592} & 1 & 2352029 & PEABIRU & 527 & 1599,0 & 1091,2 & 2331,8 \\
& & 2 & 2550045 & TEIXEIRA SOARES & 950 & 1582,8 & 927,1 & 2507,6 \\
\multirow{2}{*}{ GRUPO 3 } & \multirow{2}{*}{1844,5} & 1 & 2551035 & CAMPO DE DENTRO & 1202 & 1869,6 & 1137,8 & 2820,5 \\
& & 2 & 2553020 & FLORIANO & 481 & 1870,2 & 1097,9 & 2603,3 \\
\multirow{2}{*}{ GRUPO 4 } & \multirow{2}{*}{2060,1} & 1 & 2548000 & MORRETES & 8 & 2176,4 & 1350,8 & 2896,5 \\
& & 2 & 2652012 & VITORINO - MOINHO CAÇADOR & 710 & 2068,0 & 1240,3 & 3283,3 \\
\hline
\end{tabular}

Na Figura 3 está representado o quadro síntese para os dados mensais. Lembrando que foram utilizadas estações de diferentes regiões homogêneas, justamente para agregar a diversidade da distribuição de precipitação na área de estudo. Já que, por exemplo, um período pode ser chuvoso na Planície Costeira e seco no Terceiro Planalto. Como de fato ocorreu em janeiro de 1998, quando a estação Icatu (Terceiro Planalto) teve um acumulado pluviométrico classificado como "Seco", enquanto a estação Morretes (planície costeira) obteve a classificação de "Chuvoso". 


\section{OS DESAFIOS DA GEOGRAFIA FÍSICA NA FRONTEIRA DO CONHECIMENTO \\ Instituto de Geociências - Unicamp \\ Campinas - SP \\ 28 de Junho à 02 de Julho de 2017}

Pelo motivo de ser um quadro síntese, o qual agregou informações de diferentes estações, e também por serem 30 anos analisados, a quantificação das classes dos quantis não foi igual a da Tabela 1 em todos os períodos analisados. Para os dados mensais, as classes apresentaram as seguintes quantificações médias: muito seco $(13,3 \%)$, seco $(18,9 \%)$, normal $(28,6 \%)$, chuvoso $(18,9 \%)$, muito chuvoso $(12,8 \%)$ e misto $(7,5 \%)$.

\begin{tabular}{|c|c|c|c|c|c|c|c|c|c|c|c|c|}
\hline & JAN & FEV & MAR & $\mathrm{ABR}$ & MAI & JUN & JUL & AGO & SET & OUT & NOV & DEZ \\
\hline 1977 & $\mathrm{~N}$ & $\mathrm{~S}$ & $\mathrm{C}$ & $\mathrm{S}$ & MS & $\mathrm{N}$ & $\mathrm{S}$ & $\mathrm{N}$ & $\mathrm{S}$ & $\mathrm{S}$ & $\mathrm{C}$ & $\mathrm{N}$ \\
\hline 1978 & $\mathrm{~S}$ & MS & $\mathrm{N}$ & MS & $\mathrm{S}$ & $\mathrm{S}$ & $\mathrm{MC}$ & Misto & $\mathrm{N}$ & MS & Misto $(\mathrm{C} / \mathrm{N})$ & S \\
\hline 1979 & MS & $\mathrm{N}$ & MS & $\mathrm{N}$ & C & MS & $\mathrm{N}$ & $\mathrm{N}$ & C & $\mathrm{MC}$ & $\mathrm{N}$ & $\mathrm{C}$ \\
\hline 1980 & $\mathrm{~N}$ & $\mathrm{~N}$ & $\mathrm{~N}$ & $\mathrm{~S}$ & $\mathrm{~N}$ & $\mathrm{~N}$ & C & $\mathrm{C}$ & C & Misto & S & $\mathrm{MC}$ \\
\hline 1981 & $\mathrm{~N}$ & $\mathrm{~N}$ & S & C & MS & $\mathrm{S}$ & MS & $\mathrm{N}$ & MS & $\mathrm{C}$ & $\mathrm{N}$ & MC \\
\hline 1982 & MS & $\mathrm{N}$ & MS & MS & $\mathrm{N}$ & $\mathrm{MC}$ & MC & $\mathrm{N}$ & MS & $\mathrm{C}$ & $\mathrm{MC}$ & $\mathrm{N}$ \\
\hline 1983 & $\mathrm{~N}$ & $\mathrm{~N}$ & $\mathrm{MC}$ & MC & $\mathrm{MC}$ & $\mathrm{MC}$ & MC & MS & $\mathrm{MC}$ & $\mathrm{N}$ & $\mathrm{N}$ & S \\
\hline 1984 & $\mathrm{~N}$ & $\mathrm{~S}$ & $\mathrm{C}$ & $\mathrm{C}$ & $\mathrm{N}$ & $\mathrm{C}$ & $\mathrm{S}$ & MC & $\mathrm{N}$ & MS & $\mathrm{MC}$ & $\mathrm{C}$ \\
\hline 1985 & MS & $\mathrm{C}$ & C & $\mathrm{MC}$ & S & S & $\mathrm{S}$ & $\mathrm{S}$ & Misto & $\mathrm{S}$ & $\mathrm{S}$ & MS \\
\hline 1986 & $\mathrm{~N}$ & MC & $\mathrm{N}$ & Misto $(\mathrm{C} / \mathrm{N})$ & C & MS & MS & $\mathrm{MC}$ & $\mathrm{S}$ & S & C & $\mathrm{C}$ \\
\hline 1987 & $\mathrm{~N}$ & $\mathrm{~N}$ & $\mathrm{~S}$ & C & MC & $\mathrm{N}$ & $\mathrm{S}$ & $\mathrm{N}$ & S & $\mathrm{N}$ & Misto $(\mathrm{C} / \mathrm{S})$ & S \\
\hline 1988 & $\mathrm{~S}$ & $\mathrm{~S}$ & S & $\mathrm{C}$ & $\mathrm{C}$ & $\mathrm{N}$ & MS & MS & MS & Misto $(\mathrm{S} / \mathrm{N})$ & MS & S \\
\hline 1989 & $\mathrm{MC}$ & $\mathrm{C}$ & $\mathrm{N}$ & $\mathrm{C}$ & $\mathrm{N}$ & S & $\mathrm{C}$ & $\mathrm{C}$ & $\mathrm{C}$ & $\mathrm{N}$ & $\mathrm{S}$ & Misto \\
\hline 1990 & $\mathrm{MC}$ & MS & $\mathrm{N}$ & MC & $\mathrm{N}$ & $\mathrm{N}$ & $\mathrm{MC}$ & $\mathrm{MC}$ & C & $\mathrm{N}$ & Misto & MS \\
\hline 1991 & $\mathrm{~S}$ & MS & Misto & $\mathrm{N}$ & $\mathrm{S}$ & $\mathrm{MC}$ & MS & $\mathrm{S}$ & $\mathrm{S}$ & $\mathrm{S}$ & $\mathrm{S}$ & MC \\
\hline 1992 & MS & $\mathrm{N}$ & $\mathrm{MC}$ & Misto & $\mathrm{MC}$ & $\mathrm{S}$ & $\mathrm{C}$ & $\mathrm{C}$ & $\mathrm{N}$ & Misto $(\mathrm{S} / \mathrm{N})$ & $\mathrm{N}$ & MS \\
\hline 1993 & $\mathrm{C}$ & Misto & $\mathrm{N}$ & $\mathrm{S}$ & C & $\mathrm{N}$ & $\mathrm{C}$ & $\mathrm{S}$ & $\mathrm{MC}$ & $\mathrm{N}$ & S & Misto \\
\hline 1994 & Misto & $\mathrm{C}$ & $\mathrm{N}$ & $\mathrm{N}$ & $\mathrm{N}$ & C & $\mathrm{C}$ & MS & MS & $\mathrm{N}$ & $\mathrm{C}$ & C \\
\hline 1995 & MC & $\mathrm{N}$ & $\mathrm{C}$ & $\mathrm{N}$ & MS & $\mathrm{N}$ & $\mathrm{N}$ & $\mathrm{S}$ & $\mathrm{N}$ & $\mathrm{C}$ & S & Misto \\
\hline 1996 & $\mathrm{C}$ & $\mathrm{C}$ & MC & $\mathrm{S}$ & S & $\mathrm{N}$ & $\mathrm{N}$ & $\mathrm{N}$ & $\operatorname{Misto}(\mathrm{C} / \mathrm{N})$ & $\mathrm{MC}$ & $\mathrm{N}$ & MC \\
\hline 1997 & C & $\mathrm{C}$ & MS & MS & $\mathrm{N}$ & $\mathrm{MC}$ & $\mathrm{S}$ & C & C & $\mathrm{MC}$ & $\mathrm{C}$ & $\mathrm{N}$ \\
\hline 1998 & $\mathrm{~N}$ & $\mathrm{C}$ & $\mathrm{MC}$ & $\mathrm{MC}$ & $\mathrm{N}$ & $\mathrm{S}$ & Misto & $\mathrm{MC}$ & $\mathrm{MC}$ & $\mathrm{C}$ & MS & $\mathrm{N}$ \\
\hline 1999 & C & $\mathrm{MC}$ & $\mathrm{N}$ & $\mathrm{N}$ & $\mathrm{N}$ & C & $\mathrm{N}$ & MS & S & MS & MS & $\mathrm{N}$ \\
\hline 2000 & S & $\mathrm{MC}$ & $\mathrm{C}$ & $\mathrm{S}$ & S & C & $\mathrm{N}$ & $\mathrm{C}$ & $\mathrm{C}$ & $\mathrm{N}$ & Misto & Misto \\
\hline 2001 & Misto & $\mathrm{MC}$ & $\mathrm{N}$ & $\mathrm{N}$ & $\mathrm{N}$ & $\mathrm{N}$ & $\mathrm{N}$ & $\mathrm{N}$ & $\mathrm{N}$ & Misto & C & $\mathrm{N}$ \\
\hline 2002 & C & $\mathrm{S}$ & $\mathrm{S}$ & MS & $\mathrm{MC}$ & MS & Misto $(\mathrm{S} / \mathrm{N})$ & $\mathrm{C}$ & $\mathrm{N}$ & $\mathrm{N}$ & $\mathrm{MC}$ & Misto \\
\hline 2003 & $\mathrm{~N}$ & $\mathrm{~N}$ & $\mathrm{~N}$ & $\mathrm{~N}$ & S & $\mathrm{N}$ & $\mathrm{N}$ & $\mathrm{S}$ & Misto & Misto & Misto $(\mathrm{C} / \mathrm{N})$ & $\mathrm{N}$ \\
\hline 2004 & $\mathrm{~S}$ & $\mathrm{~S}$ & $\mathrm{~S}$ & C & C & $\mathrm{N}$ & $\mathrm{C}$ & $\mathrm{S}$ & $\mathrm{S}$ & C & $\mathrm{N}$ & S \\
\hline 2005 & C & MS & MS & $\mathrm{N}$ & $\mathrm{N}$ & $\mathrm{C}$ & $\mathrm{N}$ & $\mathrm{N}$ & MC & MC & MS & MS \\
\hline 2006 & $\mathrm{~S}$ & $\mathrm{~S}$ & $\mathrm{C}$ & $\mathrm{S}$ & MS & MS & $\mathrm{S}$ & $\mathrm{N}$ & $\mathrm{N}$ & MS & $\mathrm{C}$ & C \\
\hline
\end{tabular}

Figura 3 - Quadro síntese da caracterização da precipitação mensal.

Alguns períodos foram classificados como "Misto", pois não foi possível definir um padrão para todas as estações pluviométricas. O ano de 1983 foi o ano que mais se destacou em relação a classe "Muito Chuvoso", foram seis meses classificados dessa forma. O ano de 2001 apresentou onze meses classificados como "Normal", podendo ser considerado um ano padrão normal. Já o ano de 1988, apresentou quatro meses classificados como "Muito Seco", e quatro meses classificados como "Seco", podendo ser considerado um ano padrão seco.

Analisando os dados em relação à evolução temporal, de 1977 a 2006, observa-se, por exemplo, que o mês de janeiro teve uma ocorrência de meses chuvosos a partir do ano de 1989. No mês de fevereiro, a ocorrência de meses chuvosos se deu a partir de 1985, sendo que de 2002 a 2006 a maior parte dos anos foram secos. 


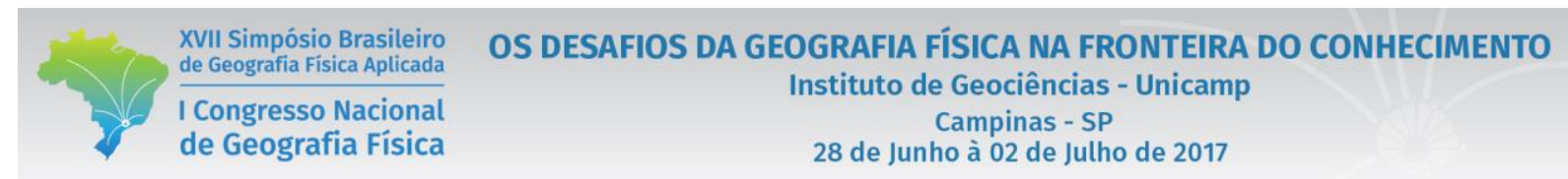

Na Figura 4 está representado o quadro síntese para os dados sazonais e anuais. Para os dados sazonais, as classes apresentaram as seguintes quantificações médias: muito seco (15\%), seco (20\%), normal (29,2\%), chuvoso (18,3\%), muito chuvoso $(13,3 \%)$ e misto $(4,2 \%)$. Para os dados anuais, as classes apresentaram as seguintes quantificações: muito seco $(13,3 \%)$, seco $(16,7 \%)$, normal $(33,3 \%)$, chuvoso $(16,7 \%)$, muito chuvoso $(13,3 \%)$ e misto $(6,7 \%)$.

\begin{tabular}{|c|c|c|c|c|c|}
\hline & VERÃO & OUTONO & INVERNO & PRIMAVERA & ANUAL \\
\hline 1977 & $\mathrm{~N}$ & $\mathrm{~S}$ & $\mathrm{~N}$ & S & $\mathrm{S}$ \\
\hline 1978 & MS & MS & $\mathrm{N}$ & $\mathrm{S}$ & MS \\
\hline 1979 & $\mathrm{~S}$ & $\mathrm{C}$ & $\mathrm{S}$ & $\mathrm{C}$ & $\mathrm{N}$ \\
\hline 1980 & Misto $(\mathrm{C} / \mathrm{N})$ & Misto & C & $\mathrm{N}$ & $\mathrm{N}$ \\
\hline 1981 & $\mathrm{C}$ & $\mathrm{S}$ & MS & $\mathrm{S}$ & $\mathrm{S}$ \\
\hline 1982 & $\mathrm{~S}$ & MS & $\mathrm{MC}$ & $\mathrm{MC}$ & $\mathrm{C}$ \\
\hline 1983 & $\mathrm{~N}$ & $\mathrm{MC}$ & $\mathrm{MC}$ & $\mathrm{MC}$ & $\mathrm{MC}$ \\
\hline 1984 & $\mathrm{~N}$ & $\mathrm{~N}$ & $\mathrm{C}$ & $\mathrm{N}$ & $\mathrm{N}$ \\
\hline 1985 & S & $\mathrm{C}$ & MS & MS & MS \\
\hline 1986 & $\mathrm{C}$ & $\mathrm{C}$ & $\mathrm{S}$ & $\mathrm{S}$ & $\mathrm{N}$ \\
\hline 1987 & S & $\mathrm{MC}$ & $\mathrm{N}$ & S & Misto \\
\hline 1988 & MS & $\mathrm{C}$ & MS & MS & MS \\
\hline 1989 & $\mathrm{MC}$ & $\mathrm{N}$ & $\mathrm{C}$ & $\mathrm{S}$ & $\mathrm{C}$ \\
\hline 1990 & $\mathrm{~N}$ & $\mathrm{C}$ & $\mathrm{MC}$ & $\mathrm{N}$ & $\mathrm{MC}$ \\
\hline 1991 & $\mathrm{~S}$ & $\mathrm{~S}$ & $\mathrm{~N}$ & MS & $\mathrm{S}$ \\
\hline 1992 & MS & $\mathrm{MC}$ & $\mathrm{C}$ & MS & $\mathrm{C}$ \\
\hline 1993 & $\mathrm{C}$ & $\mathrm{N}$ & $\mathrm{N}$ & $\mathrm{N}$ & Misto \\
\hline 1994 & $\mathrm{C}$ & $\mathrm{N}$ & $\mathrm{N}$ & $\mathrm{N}$ & $\mathrm{N}$ \\
\hline 1995 & $\mathrm{MC}$ & S & S & $\mathrm{N}$ & $\mathrm{N}$ \\
\hline 1996 & MC & $\mathrm{N}$ & $\mathrm{S}$ & $\mathrm{C}$ & C \\
\hline 1997 & $\mathrm{MC}$ & MS & $\mathrm{MC}$ & $\mathrm{MC}$ & $\mathrm{MC}$ \\
\hline 1998 & $\mathrm{~N}$ & $\mathrm{MC}$ & $\mathrm{C}$ & $\mathrm{C}$ & $\mathrm{MC}$ \\
\hline 1999 & $\mathrm{C}$ & $\mathrm{N}$ & $\mathrm{N}$ & MS & S \\
\hline 2000 & Misto & S & $\mathrm{N}$ & $\mathrm{C}$ & $\mathrm{C}$ \\
\hline 2001 & Misto $(\mathrm{C} / \mathrm{N})$ & $\mathrm{N}$ & $\mathrm{C}$ & Misto & $\mathrm{N}$ \\
\hline 2002 & $\mathrm{~N}$ & $\mathrm{~N}$ & $\mathrm{~S}$ & $\mathrm{C}$ & $\mathrm{N}$ \\
\hline 2003 & $\mathrm{~N}$ & $\mathrm{~S}$ & S & $\mathrm{N}$ & S \\
\hline 2004 & MS & $\mathrm{C}$ & $\mathrm{N}$ & $\mathrm{N}$ & $\mathrm{N}$ \\
\hline 2005 & MS & $\mathrm{N}$ & $\mathrm{N}$ & $\mathrm{MC}$ & $\mathrm{N}$ \\
\hline 2006 & $\mathrm{~S}$ & MS & MS & $\mathrm{N}$ & MS \\
\hline
\end{tabular}

Figura 4 - Quadro síntese da caracterização da precipitação sazonal e anual.

A partir da Figura 4 é possível verificar como as estações do ano ficaram classificadas intra-anualmente, e a partir disso mensurar o resultado para o ano. Porém, como a análise dos quantis foi realizada com base nos valores totais anuais, as classes se diferiram um pouco. Como exemplo, adotou-se o ano de 1998, onde apenas a estação de outono foi classificada como "Muito Chuvoso", e as estações de inverno e primavera como "Chuvoso", ou seja, a maioria; porém o ano foi classificado como "Muito Chuvoso" devido à magnitude do acumulado anual.

Zandoni (2009), em seu estudo sobre as chuvas na bacia do Paraná (1976-2005), que engloba a maior parte do território paranaense, classificou a precipitação anual a partir de duas técnicas: desvio quartílico e fórmula de Sturges. Comparando-se os resultados encontrados pelo autor com os deste trabalho, para o 


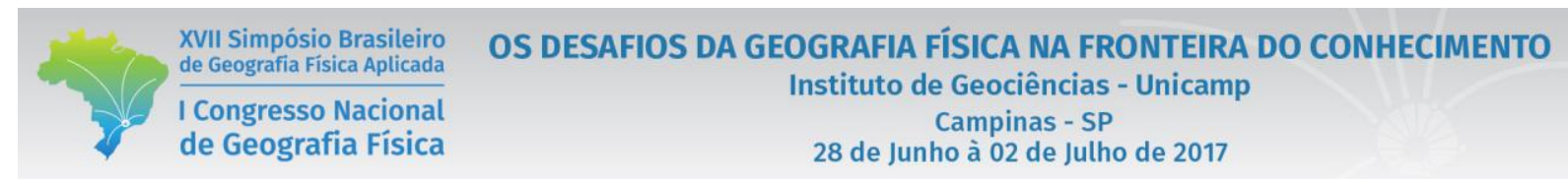

mesmo período, 58,6\% apresentaram classe semelhante. E os anos classificados como "Muito Chuvoso" e "Muito Seco" neste trabalho, apresentaram classe correspondente no trabalho do autor, que utilizou as classes: seco, habitual, chuvoso e misto. Apenas o ano de 1990, aqui classificado como "Muito Chuvoso", para Zandoni foi classificado como misto.

Os dendogramas auxiliaram na classificação de períodos que se mostraram distintos entre as estações pluviométricas, e também como forma de validar os resultados encontrados pela análise dos quantis. A Figura 5 traz o dendograma gerado para o período anual, onde é possível observar, por exemplo, que a maior distância euclidiana encontrada foi entre os anos considerados extremos, 1998 e 1983, do restante da amostra. A figura também destaca os anos de 1985, 1988 e 2006, os quais formaram um grupo se considerada a linha de corte na distância 600; e ambos foram classificados como "Muito Seco" pela técnica dos quantis.

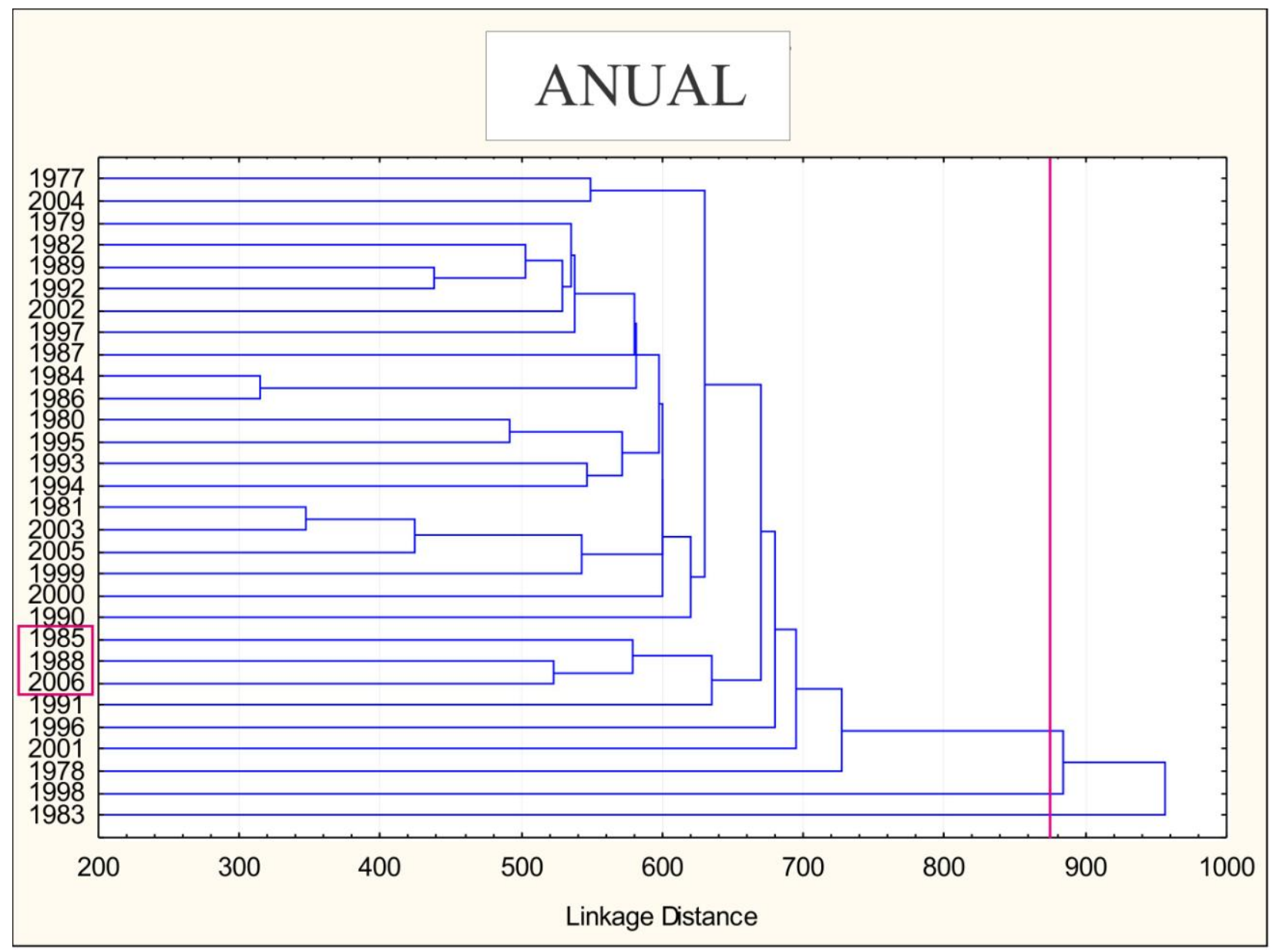

Figura 5 - Dendograma dos dados de precipitação das estações representativas de cada região homogênea, para o período anual. 
Os anos de 1983, 1998, 1997 e 1990 foram considerados "Muito Chuvosos"; já os anos de 1985, 1978, 1988 e 2006, foram considerados "Muito Secos". Os anos mencionados estão ordenados de forma decrescente para os muito chuvosos, ou seja, 1983 foi o ano que mais choveu em média, seguido por 1998, e assim sucessivamente, e os anos muito secos estão ordenados de forma crescente, ou seja, 1985 foi o ano com a menor média de precipitação.

O ano de 1983 apresentou as estações do ano outono, inverno e primavera, e os meses de março a julho e setembro classificados como "Muito Chuvoso".

O ano de 1998 apresentou a estação do ano outono, e os meses de março, abril, agosto e setembro classificados como "Muito Chuvoso".

O ano de 1997 apresentou as estações do ano verão, inverno e primavera, e o mês de outubro classificados como "Muito Chuvoso".

O ano de 1990 apresentou as estações do ano verão, inverno e primavera, e os meses de janeiro, abril, julho e agosto, classificados como "Muito Chuvoso".

O ano de 1985 apresentou as estações do ano inverno e primavera, e os meses de janeiro e dezembro classificados como "Muito Seco".

O ano de 1978 apresentou as estações do ano verão e outono, e os meses de fevereiro, abril e outubro classificados como "Muito Seco".

O ano de 1988 apresentou as estações do ano verão, inverno e primavera, e os meses de julho a setembro e novembro classificados como "Muito Seco".

Por fim, o ano de 2006 apresentou as estações do ano outono e inverno, e os meses de maio, junho e outubro classificados como "Muito Seco".

Observou-se pelas análises que o valor médio dos períodos analisados se aproximou mais do $\mathrm{Q}_{0,65}$ do que do $\mathrm{Q}_{0,35}$, sendo estes valores os limites da classe normal, isso ocorreu principalmente para os dados mensais. Em 5\% dos casos o valor médio foi superior ao quantil 0,65 (todos os casos na categoria de análise mensal). Faz-se esse parêntese com o objetivo de mostrar que utilizando este método classificatório, a média não está necessariamente inserida na classe normal.

Segundo Tavares (1976, p.81), "um ano padrão "normal", seria aquele em que a distribuição da precipitação anual de um determinado lugar fosse semelhante à distribuição das precipitações médias, obtida através de vários anos para este local”. Com as análises obtidas neste trabalho, a palavra semelhante inserida na citação do autor, poderia ser interpretada apenas como parecida, e não igual. 
Porém o autor está falando sobre a precipitação anual, e para esta categoria de análise, em todos os casos neste trabalho a precipitação média esteve inserida na faixa normal.

Se comparado o quadro síntese da classificação das precipitações, com a classificação a partir dos valores médios, a diferença dos resultados foi de $20 \%$.

\section{Conclusão}

Pelo fato de a análise ter sido realizada com base em diferentes estações pluviométricas - unindo as diferenças da distribuição pluviométrica das regiões homogêneas, a classificação da precipitação foi um tanto influenciada pelas análises e interpretações dos pesquisadores. Ou seja, com a mesma série histórica, dependendo do intérprete o resultado poderia ser um pouco diferente.

Ficou evidente que se fosse trabalhado apenas com os valores médios, os resultados seriam diferentes dos encontrados nesta pesquisa. Um ponto negativo de se utilizar os valores médios é que as diferenças das regiões homogêneas de precipitação não seriam bem representadas. Porém, a análise se torna mais fácil e rápida.

O método dos quantis, de modo geral, apresentou bons resultados, a crítica que se faz é que, por exemplo, se em janeiro de 2017 chovesse $300 \mathrm{~mm}$, e o $\mathrm{Q}_{0,65}$ fosse 299,5, o período seria considerado chuvoso, e se em 2018 chovesse 299 mm, o período seria considerado normal. Ou seja, as precipitações seriam bem próximas, porém cada período se classificaria de uma maneira. Por isso, se faz importante analisar paralelamente os totais pluviométricos para cada período estudado e também o dendograma.

\section{Bibliografia}

FRITZSONS, E.; MANTOVANI, L. E.; WREGE, M. S.; NETO, A. C. Análise da pluviometria para definição de zonas homogêneas no Estado do Paraná. RA'E GA, v. 23, p. 555-572, 2011.

GUIMARÃES, M. J. M.; LOPES, I. Análise da precipitação do município de Cruz das Almas através da técnica de quantis. XXV Congresso Nacional de Irrigação e Drenagem, São Cristóvão-SE. Novembro, 2015.

MONTEIRO, C. A. A frente polar atlântica e as chuvas de inverno na fachada sul-oriental do Brasil. USP/IG, São Paulo, 1969. 68p.

PINKAYAN, S. Conditional probabilities of ocurrence of Wet and Dry Years Over a Large Continental Area. Colorado: State University, Boulder-Co, 1966. (Hidrology papers, n. 12).

SANTOS, A. P. P. dos; ARAGÃO, M. R. da S.; CORREIA, M. de F.; SANTOS, S. R. Q. dos; SILVA, F. D. dos; ARAÚJO, H. A. de. Precipitação na cidade de Salvador: variabilidade temporal e classificação em quantis. Revista Brasileira de Meteorologia, v.31, n.4, out./dez., 2016. 
SILVA, J. U. de L. A dinâmica atmosférica e a distribuição das chuvas na região "oriental” paulista. Tese de Doutorado (Doutor em Ciências). Universidade Estadual Paulista, Rio Claro, 1999.

TAVARES, A. C. Critérios de escolha de anos padrões para análise rítmica. Revista Geografia, AGETEO, Rio Claro, ${ }^{\circ} 1$, p.79-87, 1976.

ZANDONADI, L. As chuvas na bacia do Paraná: aspectos temporais, espaciais e rítmicos. Dissertação de Mestrado (Mestre em Geografia). Universidade Estadual Paulista, Rio Claro, 2009.

ZAVATTINI, J. A.; BOIN, M. N. Climatologia geográfica: teoria e prática de pesquisa. Campinas, SP: Editora Alínea, 2013. 